\title{
Different Dental Aging Charts or Atlas Methods Used for Age Estimation-A Review
}

\author{
Dr.Vidhya Arumugam ${ }^{1} \&$ Dr.Nagabhushana Doggalli ${ }^{2} *$ \\ ${ }^{1}$ Post Graduate Student, JSSAHER, JSSDC\&H, Mysuru.Email: drvidhya86@gmail.com \\ ${ }^{2 *}$ Reader, Dept. of Oral Medicine and Radiology, JSSAHER, JSSDC\&H, Mysuru. Email: dr.nagabhushand@jssuni.edu.in
}

The literature search for articles written in the English language in PubMed, MEDLINE, Embase, Scopus and Google Scholar database by using MeSH terms Atlas methods, charts for age estimation, Schematic representation for age estimation. Age estimation plays an important role in forensic medicine, clinical dentistry, paediatric endocrinology, and archaeology. Age estimation is of wider importance in forensic medicine, not only for the purpose of identifying deceased victims but also in connection with crimes and accidents. Estimation of age by examining the living or skeletal and cadavers remains of an individual or population group has been a conventional primary identification process over the years. In mas. disaster situations, the need for an accurate, reliable, cheap, fast and easy-to-use method is imperative for the victim identification process, especially when the lack of personnel or resources dictates the help of untrained volunteers. In these cases, using a comparison method in the form of an Atlas or a chart diagram or Atlas software with the radiograph of developing teeth that would give an estimate of chronological age would be ideal. Hereby it explains the broad view of Atlas methods used for age estimation.

Keywords: Atlas methods, Age estimation charts, Schemas of age estimation, Schour and Masseler method, Ubelaker method, Alqahtani London atlas method.

\section{Introduction}

Knowing your age is a basic human right and having it documented is what gives identity to the individual (UNICEF, 1989) [1]. In the society we live in, one's date of birth is the epitome of one's entity. In other words it drives the journey of life from birth to death. Only half of the children under five years old in the developing world have their births registered. In sub-Saharan Africa 64\% of births go unregistered, and in South Asia 65\% of all births go unregistered (UNICEF, 2010:44). The implications for children can be monumental. Their official "invisibility" increases their vulnerability and the risk that violations of their rights will go unnoticed. For example, without documents to prove their age, children are more vulnerable to underage recruitment into fighting forces, to being exposed to hazardous forms of work, to early marriages. They are also more vulnerable to being treated as an adult rather than a child or juvenile in criminal proceedings, and when seeking international protection as asylum seekers $[2,3,4,5]$.

\section{Chronology of Age}

Age is determined by the date of birth and the period of time or number of years elapsed after that to any point of time, which is then called the chronological age. (Krogman, 1968; Kraemer et al., 1985). It is documented ideally in a birth certificate, hospital records, governmental databases and many more; but in the absence of these documents, other ways to establish age are important [3].

\section{Importance of Estimation of Age}

The human dentition develops over nearly a third of the average human life, with easily detected and predictable stages. Teeth also survive inhumation very well because of their minimal organic content, which is only $4 \%$ in dental enamel. They are very stable and minimally affected by environmental factors, socioeconomic status, 
Asian Journal of Basic Science \& Research Volume 2, Issue 3, Pages 64-74, July-September 2020

nutrition, dietary habits and even by endocrine factors (Garn et al., 1965a, b; Voors, 1973; Demirjian et al., 1985; Krogman and Iscan, 1986; Hillson, 1996; Gutierrez-Salazara and Reyes-Gasgaa, 2003; Aka et al., 2009) [3, 5].

The need to know the ages of unidentified asylum seekers, to make sure they are who they claim to be, is not only for the benefit of the hosting country. It is also to protect them from sexual abuse, being taken advantage of, and getting appropriate support. Developed countries also sometimes need to age their own citizens who were not recorded at birth, had their documents stolen or who have been kidnapped at some point. So an easy-to-apply method for age estimation is vital to minimize the time needed for processing cases and training personnel $[3,6]$.

The age of criminal responsibility can be as young as 7 years (in India, Switzerland and South Africa) to as old as 18 years (in Belgium and the United States). Therefore, knowing the right age in the absence of documents can be life-changing for a young person. [3,5].

\section{Dental Age Assessment [3]}

Dental age assessment can be done in an invasive or non-invasive way.

\section{Non-invasive methods/Radiographic method}

\section{Sequential tooth eruption and/or emergence}

(Demirjian, 1973; Carvalho et al., 1989; Nystrom et al., 2001; Foti et al., 2003; Moslemi, 2004; Franchi et al., 2008; Olze et al., 2008; Aggarwal 2011; Feraru et al., 2011).

\section{Development by means of calcification and/or root maturation}

Developmental schemas/ Atlas (Schour and Massler, 1941; Gustafson and Koch, 1974; Ubelaker, 1978; Kahl and Schwarze, 1988a; AlQahtani et al., 2010).

\section{Age Estimation Using Atlas or Charts Prepared from Population Surveys}

There are numerous studies in this subject which are conducted on different populations. Data derived from these studies are given in the form of charts based on eruption of deciduous teeth, shedding of deciduous teeth and eruption of permanent teeth.

Radiographical evidence of formation of crowns and root completion has been utilized for the same purpose. These charts are based on dental surveys of cross sections of the population and show the progressive states of dental development for each year of age [7].

\section{Construction of the "Tooth Development Diagram"}

It was thought being of primary interest to use only well-defined and readily identified stages of tooth development. Therefore data on timing of the following stages were collected for all teeth in both deciduous and permanent dentition, with exception of the third permanent molar [8].

(1) Commencement of mineralisation, (2) Completion of crown, (3) Eruption (the cusp(s) penetrating the gingiva) and (4) Completion of root(s). 
Asian Journal of Basic Science \& Research Volume 2, Issue 3, Pages 64-74, July-September 2020

The diagram is based on data from the following studies: Röse (1909), Cohen (1928), Logan and Kronfeld (1933), Klein et al. (1937). Schour and Massler (1941), Robinow et al. (1942), Kranz (1946), Orban 1957, Dahlberg and Maunsbach (1948), Stones et al. (1951), Clements et al. (1953), Gödény (1955), Tegzes (1959), Nolla (1960), Fanning (1961), Sjöberg (1961), Carr (1962), Moyers (1963), Lysell et al. (1964) and Haavikko (1970) [8].

\section{Different Atlas Techniques}

\section{$>$ Development of the Dental Aging Charts}

The first chronologies of dental formation can be attributed to Legros and Magitot (1880, 1881; quoted from Smith, 1991:145). These authors developed tables that displayed the appearance of dental tissues and structures for both the deciduous and permanent teeth, with emphasis on prenatal formation. . Later developmental charts appeared in American dental journals in 1883 (Black) and 1884 (Peirce), without any description of the methods or sample subjects and further modified by Black (2012) [9].

In 1933, Logan and Kronfeld examined children with various dental abnormalities, including cleft palate and linear enamel hypoplasias, and discussed the errors found in Legros and Magitot's chart of calcification. The authors found that within the table, all permanent teeth, from central incisor to second premolar in both upper and lower jaws, are shown as beginning calcification at one month after birth, whereas in reality there are differences of almost two years between formation of the dentin of the central incisor and that of the second bicuspid (1933:392) $[3,5,6,10]$.

Logan and Kronfeld (1933), also found that although the chart contains many inaccuracies, the table has for four decades been copied, without modification, by accepted authorities on histology. Smith (1991) cites the errors found in Legros and Magitot's chart as a result of their data being poorly translated into English and their table of development being partially misprinted. Smith (1991:146), however, believes that these charts were a product of each author's analyses of hypoplastic banding and found that when measured against modern studies, all are quite inaccurate. Later, in 1924, W.J. Brady printed a chart that pictographically depicts stages of dental development, eruption, and absorption that he developed after more than 25 years of study. He believed that it was a dentist's duty to know the average time of loss of the deciduous teeth. The study by Logan and Kronfeld in 1933 was a cross sectional study using histological sectioning and radiographic examination of $0-15$ year olds. The study was based on the examination of 25 fresh post-mortem specimens, however, 19 of these were under 2 years of age. From the data the authors published a detailed account of the positioning and relative development of the human dentition. $[11,12,13]$.

The most commonly used dental age estimation atlas techniques are those of Schour and Massler (1941), Moorrees and colleagues (1963), Gustafson and Koch (1974), and Anderson et al. (1976). These techniques are based on morphologically different stages of mineralization of all teeth (Willems 2001). Schour and Massler (1941) proposed 20 stages of tooth development from 4 months until 21 years of age, while Moorrees et al., (1963) proposed dental maturation of permanent teeth (maxillary and mandibular incisors) divided into 14 stages, from initial cusp formation to apical closure for both gender. Gustafson and Koch's (1974) technique proposed tooth 
development from mineralization, completion of crown formation, eruption, to completion of root formation [14-16].

In 1941, Schour and Massler published an important study that summarised the development of the human dentition in an 'atlas style' chart. It was based on the data collected by Logan and Kronfeld and other histological studies of the developing dentition undertaken by Schour around the same time. The summary of this information into a pictorial or 'atlas type' chart provided the profession with a tool that would not only be used in every day practice, but would also prove useful in estimating the age of an individual in a forensic context $[3,14,17]$.

The 1944 Schour and Massler chart was printed in their Atlas of the Mouth and Adjacent Parts in Health and Disease (1958) published by the American Dental Association, and includes the same error ranges. This version of their chart is very similar to their 1944 chart with the first difference being seen at age 6 , with development of the permanent upper and lower canines beginning root initiation. Root initiation has not begun in the previous chart at this age and does not reach the same stage in the 1958 chart until 8 years of age. At 7 years in the 1958 chart, the development of the permanent lower canine is shown with the development of the root half shaded. This seems to be the author's way of displaying the high variability in the formation of this particular tooth. At age 8 , the stages again become similar in their formation to the 1944 chart [17].

In 1988, Kahl and Schwarz process the data of Schour and Massler and show a slowdown in the development of the permanent dentition among the selected cohort group used in their studies. Another scientific study led to the reworking of the Schour and Massler diagrams were conducted by Ubelaker in 1978, which developed diagrams based on his Indian studies. These diagrams are included in the "Recommendations for Age and Sex Diagnoses of Skeletons" in 1980 [13].

Ubelaker's 1989 explained, Dental Aging Chart from Human Skeletal Remains, Reprinted with permission of Taraxacum Inc. Publishing Company and Douglas H. Ubelaker. The library at the American Dental Association in Chicago was unable to locate an earlier edition of the wall-sized chart and the only other versions of the Schour and Massler chart were published by the Journal of the American Dental Association in 1940 and 1941 [5,17,18].

Ubelaker 2002: as in 1978, and again in 1989, Ubelaker expanded and changed many of the error ranges originally given by Schour and Massler in 1944. This change seems to be primarily due to the fact that Ubelaker's chart was developed for analysis of Native American Indians and other non-white dentitions after determining that Schour and Massler's chart consistently overestimated the ages at death of these individuals and underestimates their actual growth rate (Merchant and Ubelaker, 1977). Despite the modifications made for Native American individuals, Ubelaker's chart has now become a recognized world standard for all ancestral groups [17].

The advantages of using this system to estimate the age of an individual are that it is non-destructive, being able to conduct the assessment on radiographs of the jaws; it is simple and does not require any specialised training to recognise specific stages of development, as required in many of the more recently developed systems; and it does not require the use of specialized equipment beyond an X-ray apparatus. [3,5,17]. The last method explored in the current research is the London Atlas method put forward by Dr.Sakher J. AlQahtani and colleagues in 2008, 
then revised in 2010. AlQahtani and his colleagues looked at two different ethnic groups for their research, half of the subjects were of European Ancestry and half were Bangladeshi. The London Atlas chart was developed to show the growth and emergence of teeth for individuals anywhere from 28 weeks in utero to those 23 years of age [10]. To create the chart the researchers looked at 704 radiographs of individuals of known age. The diagrams were meant to show the median tooth development and the alveolar eruption stages. This chart is divided into different sections based on development. In the last trimester of pregnancy, diagrams represent monthly development, two weeks apart when you get to 40-week mark, quarterly for the individual's first year, and yearly after that. One thing the author wanted to point out, especially for this study, was that birth was not an age but rather an event that does not affect the dental formation (AlQahtani et al. 2010). A benefit to using this method is that the chart is freely available on the Internet for public use [18].

Tooth formation stages were then created using transparent tracing paper over the full ideal tooth form drawn previously by the examiner (SA). The outlines of the developmental stages based on Moorrees' stages (Moorrees, Fanning et al., 1963a; b) [15].

Eruption of teeth through the alveolar bone was assessed according to modified Bengston's stages (Bengston, 1935; Liversidge, 2001) and was replicated in the diagrams in relation to a black line representing the alveolar bone. After all teeth were assessed, the median developmental stages were identified for each tooth for every age category and were used to illustrate diagrams for each chronological year for males, females and for mixed sex. A midway point was selected to be at 6 months of every chronological year with a range of plus and minus 6 months The first year of life was represented by two diagrams, midpoint at three and nine months [19].

The Atlas of tooth development and eruption has been designed and published in the American Journal of Physical Anthropology (AlQahtani et al., 2010) and is available to download for free through the Institute of Dentistry's website: www.atlas.dentistry.qmul.ac.uk in 17 languages: Arabic, traditional Chinese, Simplified Chinese, Dutch, English, Farsi, French, German, Greek, Hindi, Japanese, Malay, Portuguese, Romanian, Russian, Spanish and Urdu. It has been used in many workshops, incorporated into several universities' curricula around the world and adopted by several forensic societies [18].

According to Jane Taylor, Age estimation charts for a modern Australian population Matthew Blenkin was published in Forensic Science International in 2012 [20].

Calculation of the biological age of an individual has application in many fields of dentistry. It can be used to determine the appropriate timing of interventionist treatment for example in orthodontics; to analyse the developmental stage of an individual relative to the general population in the management of genetic or congenital conditions which disturb growth; and to estimate the age of a living or deceased person for forensic purposes. Many of the techniques used to estimate age can be quite time consuming to complete.

This project reinterpreted the Australian data previously collected by Blenkin and other relevant studies and applied it to a schematic similar to that of Ubelaker to develop a reliable, convenient and contemporary reference for use in age estimation [20]. 
According to Beytullah Karaday, Development of dental charts according to tooth development and eruption for Turkish children and young adults was published in Imaging Science in Dentistry 2014 [21].

In this study, they aimed to develop dental charts for Turkish children and young adults of both genders within the age group of 4.5-22.5 years according to tooth mineralization and eruption in a format similar to that proposed by AlQahtani et al. In total, 753 digital panoramic radiographs from 350 males and 403 females were assessed. The permanent teeth were evaluated according to the classification system described by Demirjian et al. The eruption stage was assessed with Bengston's system, which was modified by AlQahtani et al at four points.

The dental charts presented here included information that could be beneficial to dental clinicians in making appropriate diagnosis and planning orthodontic and surgical procedures. These charts also provided datasets for preliminary dental age estimation in Turkish children and young adults [21].

According to Shalmira Karkhanis, Daniel Franklin, Forensic Anthropology Population Data Dental age estimation standards for a Western Australian population was published in Forensic Science International in 2015 [22].

A total of 392 digital orthopantomograms (202 male and 190 female) of Western Australian individuals are analyzed. Following, Moorrees et al. (1963a) dental development and root resorption was assessed. Alveolar eruption was analyzed following Bengston (1935). Stages of dental development were used to formulate a series of age estimation polynomial regression models; prediction accuracy ( 0.998 to 2.183 years) is further validated using a cross-validation (holdout) sample of 30 film orthopantomograms. A visual atlas of dental development and eruption was subsequently designed for the pooled sex sample. The standards presented here represent a non-invasive and statistically quantified approach for accurate dental age estimation in Western Australian juvenile individuals [22].

\section{Limitations of Atlas Methods}

The common drawbacks of the previous schemas are the lack of uniform age distribution and/or the limited age range that fails to cover the entire developing dentition. A uniform age distribution with similar numbers for each year of age improves variance across the age range (Bocquet-Appel and Masset, 1982; Konigsberg and Frankenberg, 2002). Whereas a normal age distribution has high precision around the mean value but with low precision at the age extremes.

Other limitations are the lack of clarity in identifying crown and root developmental stages as almost all of these schemas were based on dental radiographical description of tooth development directly or indirectly, yet they presented anatomical drawings, concealing the internal tooth developmental stages [5].

When assessing tooth development from dental radiographs, one can distinguish between consecutive developmental stages more easily using internal hard tissues, such as the shape of the pulp chamber or root canal, improving sensitivity and performance measures (Moorrees et al., 1963a, b; Demirjian, 1973; Haavikko, 1974), yet no schematic method delivers that. All the previously mentioned schemas used anatomical representations of teeth 
that mask internal tooth structures and with no information regarding eruption reference, with the exception of Ubelaker (1978), who used gingival emergence as a reference $[5,15,18]$.

\section{Merits of Atlas Method}

There are several methods of age estimation based on dental age, but most of them are based on formulae and lengthy techniques only a specialist can deliver (Demirjian et al., 1973; Roberts et al., 2008), sometimes using special equipment (Bauer, 2007; Heinrich et al., 2007). In mass disaster situations, the need for an accurate, reliable, cheap, fast and easy-to-use method is imperative for the victim identification process, especially when the lack of personnel or resources dictates the help of untrained volunteers. In these cases, using a comparison method in the form of an Atlas or a diagram or computer software with the radiograph of developing teeth that would give an estimate of chronological age would be ideal $[3,5]$.

\section{Discussion}

Amongst the various methods used for age determination in individuals, the radiological method has certain advantages over histological and biochemical methods. Other methods require either extraction or preparation of microscopic sections of at least one tooth from each individual; these methods therefore cannot be used in living individuals and in cases where it is not acceptable to extract teeth for religious or scientific reasons. Besides, they are quite expensive and require some sophisticated laboratory equipment. On the contrary, the radiographic method is a simple, quick, economic, non-mutilating and non-invasive method of age identification. Additionally, it can be applied for identifying the age in dead as well as living persons and in all communities [23, 24].

Age estimation is relatively difficult in pre-natal and neonatal phases compared with the post-natal phase. Though histological examination of tooth germs will be able to decide the age earlier in the pre-natal phase, it will be an invasive and time-consuming procedure. From the pre-natal dental development of the foetus until the eruption of the first tooth into the oral cavity post-Nataly, the radiological examination of the jaw bones for the developing tooth germs will be the mainstay for the age assessment [5, 23, and 24].

Similarly, in the post-natal phase, between the ages of 2.5 and 6 years i.e. up to eruption of the first permanent tooth, there is no clinical evidence available to determine the age, so radiography will play a major role in assessing the jaw bones for developing permanent dentition. The best precision and accuracy for radiographic age estimation is achieved when individual growth is rapid and many teeth are under development. After the age of 14 years, estimation becomes difficult since most of the dentition is completely developed. Various age estimation methods or dental development surveys are available, although they are different from each other. They provide us with two types of information: the sequence of developmental events and the timing at which these events occur. Most surveys indicate that the least amount of variation in development occurs least in the younger age group but it increases with age [25].

The Schour and Masseler method has some drawbacks as these charts do not have separate surveys for males and females, and the mean age range of 2-5 years is put at 6 months and is thus too narrow. Later, Ubelakar modified these charts to widen these ranges. Regardless of these drawbacks, these charts are being used more commonly in 
age determination. Although the method by Moorees et al has separate surveys for males and females, interobserver variation was found and confined to $;$ one stage, which may suggest the difficulty in identifying where one stage begins and another one ends when using a rating system with so many intermediate stages. Therefore, it is advised to rate the stages carefully or, when applicable, use other methods, such as the Demirjian method $[14,25,26$, and 27].

\section{Summary and Conclusion}

In all the methods used for age determination, the evaluation of radiographs for stages of tooth calcification is the most reliable as tooth calcification can be observed from radiographs for a period of several years and it is not altered by local factors such as lack of space or over retention of deciduous teeth. It also allows the assessment of the period when no clinical tooth emergence takes place. Additionally, age estimation would be relatively accurate since the number of teeth passing through various stages of calcification is available [26].

Various schemas have been compiled throughout the last century to show dental development. One of the first schemas to be used widely is Schour and Massler's Atlas (1941) and it has been the benchmark for the past 70 years. Gustafson and Koch (1974) used data from 20 sources combining anatomical, radiographic and gingival eruption to construct a schematic representation of tooth formation and eruption from prenatal to the age of 16 years. It illustrates the range of tooth development and the peak age for each stage.

Ubelaker's chart (1978) was loosely based on Schour and Massler's Atlas using additional North American Indian population data. Brown et al. (1985) demonstrated permanent tooth development using anatomical tooth illustrations tabulated for the ages 3 to 12 years based on Schour and Massler's Atlas. Kahl and Schwarz (1988b) updated the Atlas using 993 radiographs of children aged 5 to 24 years and produced anatomical charts for the sexes. Recently with London Atlas method by Alqahtani 2010, followed by different authors for different populations. $[5,14,18,27]$

All the past schemas cover a limited age range, except for Schour and Massler's and Ubelaker's schemas that cover dental development from prenatal to early adulthood, which made them the most wildly used ones [18]. In conclusion, radiology has become an essential tool in the processes of human age identification. There could not be rigid guidelines regarding the age estimation, hence the degree of dental certainty or probability of the findings may be specified. Use of multiple age estimation methods, multiple teeth and experienced investigators aid towards the optimal age estimation.

Different Dental Aging Charts method

\begin{tabular}{|l|c|l|}
\hline \multicolumn{1}{|c|}{ Author } & Year & \multicolumn{1}{c|}{ Method } \\
\hline Legros and Magitot & 1880 & First chronologies of dental formation in Dissection data \\
\hline Logan and Kronfeld & 1933 & $\begin{array}{l}\text { Cross sectional study using histological sectioning and } \\
\text { Radiographic examination for 0-15 years }\end{array}$ \\
\hline Schour and Massler & 1941 & $\begin{array}{l}\text { Proposed 20 stages of tooth development from 4 months } \\
\text { until 21 years of age for combined sex }\end{array}$ \\
\hline
\end{tabular}




\begin{tabular}{|c|c|c|}
\hline Schour and Massler & 1944 & $\begin{array}{l}\text { Represented } 22 \text { stages of dental development from } 5 \\
\text { months of intrauterine life up to } 35 \text { years. Classic example } \\
\text { of the commonly used atlas approach, titled "Development } \\
\text { of human dentition" reprinted by ADA }\end{array}$ \\
\hline Moorrees & 1963 & $\begin{array}{l}14 \text { stages, from initial cusp formation to apical closure for } \\
\text { both genders }\end{array}$ \\
\hline Gustafson and Koch & 1974 & $\begin{array}{l}\text { Proposed tooth development from mineralization, } \\
\text { completion of crown formation, eruption, to completion of } \\
\text { root formation in a chart }\end{array}$ \\
\hline Ubelaker & 1978 & $\begin{array}{l}\text { Reworking of the Schour and Massler diagrams, which } \\
\text { developed diagrams based on his Indian studies, These } \\
\text { diagrams are included in the "Recommendations for Age } \\
\text { and Sex Diagnoses of Skeletons" in } 1980\end{array}$ \\
\hline Brown & 1985 & $\begin{array}{l}\text { Permanent tooth development using anatomical tooth } \\
\text { illustrations tabulated for the ages } 3 \text { to } 12 \text { years based on } \\
\text { Schour and Massler's Atlas }\end{array}$ \\
\hline Ubelaker & 1987 & $\begin{array}{l}\text { Appearance and union of epiphyses, bone size, the loss of } \\
\text { deciduous teeth, the eruption of teeth, and dental } \\
\text { calcification, recommends Moorees (1963) }\end{array}$ \\
\hline Kahl and Schwarz & 1988 & $\begin{array}{l}\text { Processed the data of Schour and Massler and show a } \\
\text { slowdown in the development of the permanent dentition } \\
\text { among the selected cohort group used in their studies. }\end{array}$ \\
\hline Kahl and Schwarz & $1988 b$ & $\begin{array}{l}\text { Updated the Atlas using } 993 \text { radiographs of children aged } \\
5 \text { to } 24 \text { years and produced anatomical charts for the sexes }\end{array}$ \\
\hline Ubelaker & 1989 & $\begin{array}{l}\text { Dental Aging Chart from Human skeletal remains, } \\
\text { reprinted with permission of Traxacum. }\end{array}$ \\
\hline Ubelaker & 1999 & Representation of Schour and Massler's Atlas \\
\hline Sakher J.AlQahtani et al. & $\begin{array}{l}2008 \& \text { \& } \\
\text { revised in } \\
2010\end{array}$ & $\begin{array}{l}\text { London Atlas method, } \\
\text { Growth and emergence of teeth for individuals anywhere } \\
\text { from } 28 \text { weeks in utero to those } 23 \text { years of age (European } \\
\text { Ancestry and half were Bangladeshi), Outlines of the } \\
\text { developmental stages based on Moorrees' stages (1963a) } \\
\text { Eruption of teeth through the alveolar bone was assessed } \\
\text { according to modified Bengston's stages (1935) }\end{array}$ \\
\hline Matthew Blenkin & 2012 & $\begin{array}{l}\text { Age estimation charts for a modern Australian population, } \\
\text { for male and female in the convenient Schour and Massler } \\
1944 \text { format }\end{array}$ \\
\hline Beytullah Karaday & 2014 & $\begin{array}{l}\text { Dental charts according to tooth development and eruption } \\
\text { for Turkish children and young adults, tooth mineralization } \\
\text { and eruption in a format similar to that proposed by } \\
\text { AlQahtani } 2010\end{array}$ \\
\hline Shalmira Karkhanis & 2015 & $\begin{array}{l}\text { Data Dental age estimation standards for a Western } \\
\text { Australian population, Analysed by following Moorrees } \\
\text { (1963), dental development and root resorption was } \\
\text { assessed. Alveolar eruption was analysed by following } \\
\text { Bengston (1935) }\end{array}$ \\
\hline
\end{tabular}


References

1. UNICEF. (1989). "Convention on the rights of the child." Retrieved 01 May, 2012, from

http://www.unicef.org/crc/

2. UNICEF. (2012). "Fact sheets: Birth registration." Retrieved 01 May, 2012, from

http://www.unicef.org/newsline/2003/03fsbirthregistration.htm

3. Catherine Adams, Romina Carabott and Sam Evans, Forensic Odontology: An Essential Guide, First Edition. 2014 by John Wiley \& Sons, Ltd.

4. Age assessment practices: a literature review \& annotated bibliography, Terry Smith, Laura Brownlees.

5. The London Atlas: developing an atlas of tooth development and testing its quality and performance measures, AlQahtani, Sakher Jaber, 2012.

6. Evidence-based Forensic Dentistry. Balwant Rai, Jasdeep Kaur., first edition, 2013.

7. Nystrom M, Peck L, Kleemola - Kujala E, Evalahti, Kataja M (2000) Age estimation in small children: reference values based on counts of deciduous teeth in Finns. Forensic Sci Int.; 110:179-88.

8. Gustafson, G. and Koch, G. (1974). "Age estimation up to 16 years of age based on dental development." Odontologist Revy 25(3): 297-306.

9. Black, S. (2012). Professor of anatomy and forensic anthropology at the University of Dundee. Dundee. Age assessment practices: a literature review \& annotated bibliography, Terry Smith, Laura Brownlees.

10. AlQahtani, S. J. (2008). Atlas of tooth development and eruption. Barts and the London School of Medicine and Dentistry. London, Queen Mary, University of London. MClinDent.

11. Logan, W. and Kronfeld, R. (1933). "Development of the human jaws and surrounding structures from birth to age fifteen." Journal of the American Dental Association 20(379).

12. Krogman, W. M. and Işcan, M. Y. (1986). The human skeleton. Forensic medicine. Charles, C. Springfield, IL, Thomas Pub.

13. Kahl, B. and Schwarze, C. (1988a). "Updating of the dentition tables of i. Schour and m. Massler of 1941." Fortschritte der Kieferorthopadie 49(5): 432-43.

14. Schour, Massler M (1941). The development of the human dentition. Journal of the American Dental Association 28: 1153-1160.

15. Moorrees, C., Fanning, E. and Hunt, E. (1963b). "Age variation of formation stages for ten permanent teeth." Journal of Dental Research 42: 1490-502.

16. Gustafson, G. and Koch, G. (1974). "Age estimation up to 16 years of age based on dental development." Odontologist Revy 25(3): 297-306. 
17. Prince DA, Ubelaker DH (2002) Application of Lamendin's adult dental aging technique to a diverse skeletal sample. J Forensic Sci 47: 107-116.

18. AlQahtani, S. J., Hector, M. P. and Liversidge, H. M. (2010). "Brief communication: The london atlas of human tooth development and eruption." American Journal of Physical Anthropology 142(3): 481-490.

19. Bengston, R. G. (1935). "A study of the time of eruption and root development of the permanent teeth between six and thirteen years ". Northwest University Bulletin 35: 3-9.

20. Blenkin, M. and Taylor, J. (2012). "Age estimation charts for a modern australian population."Forensic Science International In press (0).

21. Beytullah Karaday, Hüseyin Af in, Abdi Ozaslan1, ükriye Karaday, Development of dental charts according to tooth development and eruption for Turkish children and young adults Imaging Science in Dentistry 2014; 44: 103-13.

22. Shalmira Karkhanis, Peter Mack, Daniel Franklin, Forensic Anthropology Population Data Dental age estimation standards for a Western Australian population in Forensic Science International 257 (2015) 509.e1-509.

23. Achary AB, Sivapathasundharan B. Forensic odontology. In: Rajendran R, Sivapathasundharan B, (eds). Shafer's textbook of oral pathology (6th edn). India: Elsevier Private Ltd, 2009, pp 871-892.

24. Vandevoort FM, Bergamans L, Cleyenbreugel JV, Bielen DJ, Lambrechts P, Wevers M. Age calculation using X-ray microfocus computed tomographical scanning of teeth: A pilot study. J Forensic Sci 2004; 49: 787-790.

25. Ciapparelli L. The chronology of dental development and age assessment. In: Clark DH, (Ed). Practical forensic odontology. Oxford: Wright Butterworth-Heinemann Ltd, 1992, pp 22-42.

26. AS.Panchbhai, Dental radiographic indicators, a key to age estimation, Dentomaxillofacial Radiology (2011) 40, 199-212, The British Institute of Radiology.

27. Ubelaker, D.H. (1978). Human skeletal remains: Excavation, analysis, interpretation. Chicago, Aldine Publishing Co. Inc. 